\title{
Antisense Therapy
}

National Cancer Institute

\section{Source}

National Cancer Institute. Antisense Therapy. NCI Thesaurus. Code C16236.

Treatment using nucleic acid oligomers to bind to specific mRNA to deter protein production and alter disease process. 